\title{
Hadron form factors using density-density correlators
}

\section{Alexandrou, G. Koutsou*}

Department of Physics, University of Cyprus, CY-1678 Nicosia, Cyprus

E-mail: koutsoulucy.ac.cy

\section{H. Neff}

University College London, Center for Computational Science, 20 Gordon Street, London WC1, $U K$

Gauge invariant density-density correlators yield detailed information on hadron structure. Hadron deformation and form factors can be extracted for momentum transfers up to about $6 \mathrm{GeV}^{2}$. We use stochastic techniques and dilution to compute the all to all propagator required for the exact evaluation of density-density correlators. We present first results for the pion form factor.

XXIVth International Symposium on Lattice Field Theory

July 23-28, 2006

Tucson, Arizona, USA

\footnotetext{
* Speaker.
} 


\section{Introduction}

A number of experiments have been performed recently to determine a possible deformation in the nucleon [1]. Lattice QCD provides a model independent method for studying hadron deformation via the evaluation of density-density correlators. We show that it is now feasible to evaluate density-density correlators for mesons and baryons without any approximation. Such an evaluation requires a computation of all to all propagators, which is carried out using stochastic techniques and dilution. We show results for hadron charge distributions and demonstrate how hadron form factors can be extracted from density-density correlators. We present first results for the pion form factor.

\section{Density-density correlators}

For general time insertions the density-density correlator is given by:

$$
C\left(\vec{y}, t_{1}, t_{2}\right)=\int d^{3} x d^{3} z\left\langle h(\vec{z}, t)\left|j_{0}^{u}\left(\vec{x}+\vec{y}, t_{2}\right) j_{0}^{d}\left(\vec{x}, t_{1}\right)\right| h(\overrightarrow{0}, 0)\right\rangle
$$

where $j^{0}$ is the time ordered density operator i.e. $j_{f}^{0}(x)=: \bar{q}_{f}(x) \gamma_{0} q_{f}(x)$ : for a quark of flavor $f$, while $|h\rangle$ denotes any hadronic state. The integration over the $\vec{x}$ coordinate sets the sink momentum equal to that of the source. The integration over $\vec{z}$ sets the momentum of the source and sink to zero. These two sums require summation over both spatial coordinates of the quark propagator connecting the density insertions with the sink and thus one needs to evaluate all to all propagators.

In this work we use stochastic and dilution techniques to compute all to all propagators. It has been demonstrated [2] that an estimate for the all to all propagator can be computed by inverting the Dirac operator for an ensemble of noise vectors as sources. If these noise sources are created in such a way that they obey:

$$
\left\langle\eta_{\mu}^{a}(x) \eta_{v}^{b^{\dagger}}(y)\right\rangle_{r}=\delta(x-y) \delta_{a, b} \delta_{\mu, v} \quad \text { and } \quad\left\langle\eta_{\mu}^{a}(x)\right\rangle_{r}=0
$$

where $r$ denotes the number of noise vectors in the ensemble, then one can invert for each of these noise vectors and obtain an estimate for the all to all propagator:

$$
\left\langle\psi_{v}^{b}(x) \eta_{\mu}^{a^{\dagger}}(y)\right\rangle_{r} \rightarrow\left(M^{-1}(x, y)\right)_{v, \mu}^{b, a}
$$

where $\psi_{v}^{b}(x)=\left(M^{-1}(x, y)\right)_{v, \mu}^{b, a} \eta_{\mu}^{a}(y)$ is the individual solution for each of the $r$ noise vectors. We additionally employ a method known as dilution, which gives a better estimate for the all to all propagator [3]. The method essentially proposes a way for creating the noise source ensemble in Eq. (2.2). In this work we use even - odd, color - spin diluted noise vectors i.e. each of the $r$ noise vectors of the ensemble has random entries only on either odd or even spatial sites and on one color - spin component with all other entries set to zero. Since the time slices of the source, sink and density insertions are fixed the noise vectors are in effect already diluted in time.

\section{Wave functions}

We first consider the case where the two density insertions of Eq. (2.1) are taken at equal times. This equal time correlator, shown schematically for mesons in Fig. 1, reduces to the wave function 
squared in the non - relativistic limit. For baryons, the wave function is generally a function of two relative coordinates and thus requires the calculation of a three-density correlator depicted in Fig. 2 . In this work we compute the one particle correlator with two density insertions on two different quark lines of the baryon. This is equivalent with integrating the three-density correlator over one relative coordinate as demonstrated in Ref. [4] when the diagram in Fig. 2a was considered.

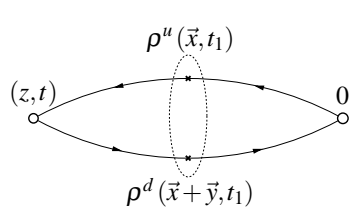

Figure 1: The density-density correlator for a meson.

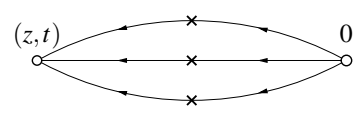

(a)

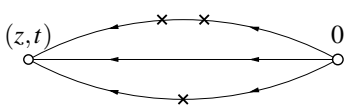

(b)

Figure 2: The three-density correlator for a baryon. The density insertions are denoted by the crosses.

In this study we use 200 unquenched gauge configurations produced by the SESAM collaboration [阿] on a lattice of size $16^{3} \times 32$, at $\beta=5.6$ and $\kappa=0.157$. Using the mass of the nucleon in the chiral limit we obtain $a^{-1}=2.56 \mathrm{GeV}$ yielding $m_{\pi} \simeq 950 \mathrm{GeV}$. We use Wuppertal smearing to construct the interpolating fields for the source and sink. HYP smearing is applied to the gauge links that enter the Wuppertal smearing function. This decreases considerably the time interval needed for the suppression of excited states and allows to place the density insertions as early as three time slices from the source [6]. Although placing the density insertions close to the source or sink is not important for the equal time correlators it is crucial for the extraction of form factors discussed in the next section (see discussion connected with Fig. 6). Similarly to satisfy the requirements for the extraction of form factors, the minimal separation between source and sink that is required is $t=14$. We take the same source-sink time separation also in the evaluation of equal time correlators so that the same set of noise vectors can be used. The evaluation of the densitydensity correlator requires a forward propagator from the source and two all to all propagators, namely one from the density insertion at $t_{1}=3$ and one from the sink. We note that the time slice of the second density insertion can be varied without additional cost a crucial observation for the evaluation of form factors from density correlators. For each stochastic inversion we use 6 sets of even - odd, color - spin diluted noise vectors. For comparison a separate computation was carried out where no summation was performed on the sink and thus the momentum of the hadronic state is not explicitly set to zero.

\subsection{Results}

In Fig. B we show the density-density correlators for the pion and the $\rho$ meson. The distributions are compared to the case where no explicit zero momentum projection is carried out. If the non-relativistic limit is a good enough approximation so that factorization of the center of mass momentum can be assumed then there should be no difference between the two evaluations. For the pion there is very little difference showing that either factorization is a good approximation or exponential suppression of the time evolution sufficiently damps out higher momentum states. On the other hand, the $\rho$ distribution is broader when explicit projection to the state of zero momentum is carried out. This shows that even for this large quark mass, factorization of the center of mass momentum does not strictly hold. These distributions are fitted using an exponential Ansatz 


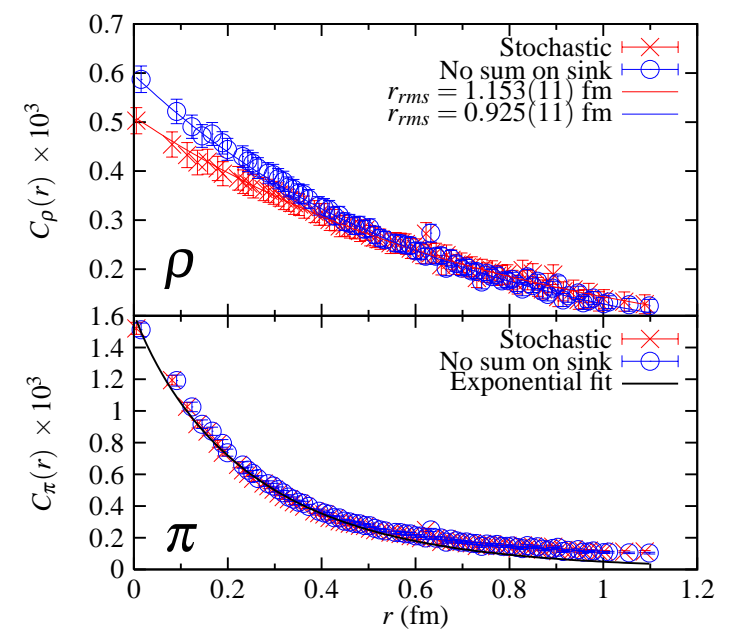

Figure 3: The correlators for the pion (bottom) and the $\rho$, in the zero-spin projection, (top). The crosses are the results of the exact evaluation and the circles are the results when no explicit zeromomentum projection is carried out.

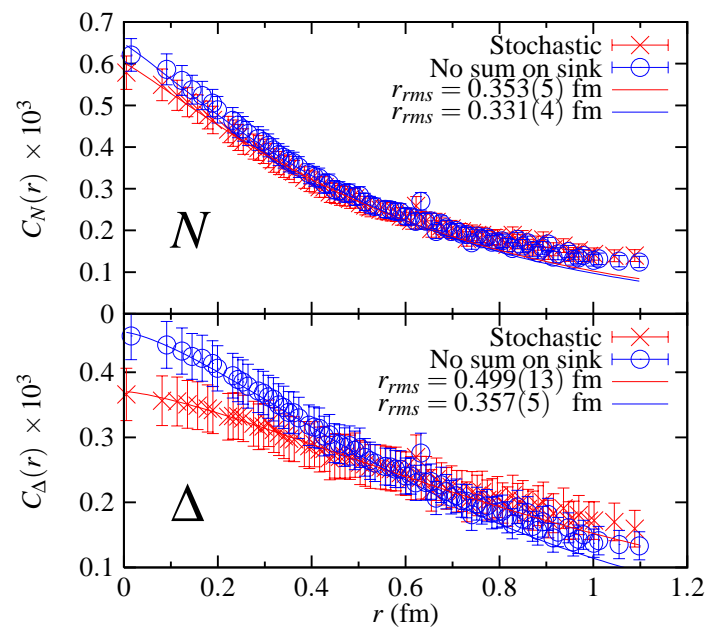

Figure 4: The correlators for the nucleon (top) and the $\Delta$ in the $\pm 3 / 2$ spin projection (bottom). The notation is the same as that of Fig. 3 .

allowing the extraction of the root mean square (r.m.s.) radii. For the pion we find an r.m.s. radius $r_{r . m . s}=0.197(2) \mathrm{fm}$ and for the $\rho$ meson $r_{r . m . s}=0.576(6) \mathrm{fm}$. The latter is approximately $25 \%$ larger as compared to what is obtained without explicit zero momentum projection.

In Fig. $甘$ we make the same comparison for the nucleon and the $\Delta$ correlators. Both states are broader when all to all propagators are used to project to zero momentum. An Ansatz that describes these distributions accurately is of the form $e^{-(x / \sigma)^{d}}$. Fits to the distributions yield $d \simeq 1.2$ for the nucleon and $d \simeq 1.5$ for the $\Delta$. The r.m.s. radius for the nucleon is only slightly bigger when the zero momentum projection is implemented whereas for the $\Delta$ we find a $30 \%$ increase.

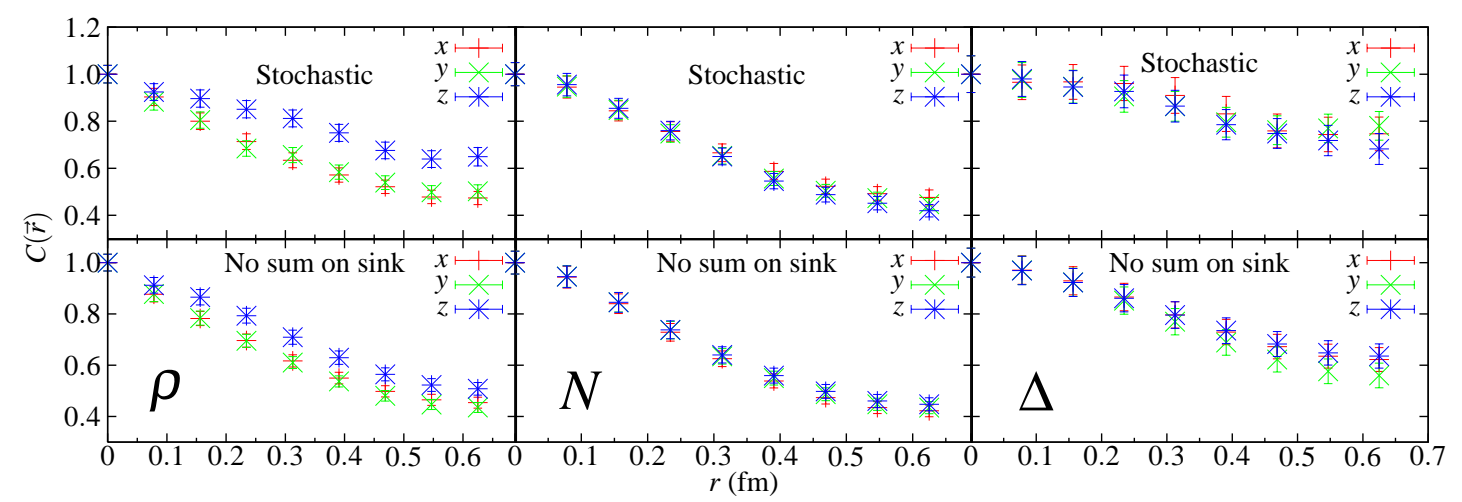

Figure 5: The $\rho$ (left), nucleon (center) and $\Delta$ (right) correlators projected along the axes. Top: with zero momentum projection. Bottom: with no zero momentum projection.

To probe hadron deformation we project our results for the density-density correlators along the spin axis (taken as the $z$ axis) and perpendicular to the spin axis. In Fig. 5 we show the projec- 
tions for the $\rho$ meson in the zero spin projection, the nucleon and the $\Delta$ in the $\pm 3 / 2$ spin projection. We again compare the exact results with the case where the summation on the sink is omitted. The $\rho$ correlator shows an elongation along the spin axis. As expected this elongation is much clearer when the state is projected to zero momentum. For the nucleon on the other hand, no deformation is detected within this method. The $\Delta$ baryon is too noisy to extract any useful information concerning a possible deformation. More statistics are clearly needed if one is to draw any definite conclusion for this hadronic state.

\section{Form factors}

The density-density correlator can be used to extract hadronic form factors 讶. Writing Eq. (2.1) on the lattice and inserting three complete sets of hadronic states, one derives the expression:

$$
\sum_{\vec{p}, n, s}\left|\left\langle\tilde{J}_{s} \mid h_{s}(0)\right\rangle\right|^{2} \frac{\left|\left\langle h_{s}(0)\left|j_{0}\right| n, \vec{p}, s\right\rangle\right|^{2}}{8 M_{h}^{2} E_{n}(p)} e^{i \vec{p} \cdot \vec{y}} e^{-E_{n}(p)\left(t_{2}-t_{1}\right)} e^{-M_{h}\left(t-\left(t_{2}-t_{1}\right)\right)},
$$

where $s$ represents internal degrees of freedom of the hadronic state and $\tilde{J}_{s}$ represents the smeared interpolating field used. In Eq. (4.1) we assume isospin symmetry $\left(j_{0}=j_{0}^{u}=j_{0}^{d}\right)$. We also assume that the time separation between the density insertions from the source and sink are large enough so that excited state contributions are suppressed. Now if, in addition, $t_{2}-t_{1}$ is large enough and we take the Fourier transform over the $\vec{y}$ coordinate we arrive at the following expression:

$$
G^{h^{0} j^{0} h}\left(s ; \vec{q} ; t_{1}, t_{2}, t\right)=\sum_{s^{\prime}}\left|\left\langle\tilde{J}_{s} \mid h_{s}(0)\right\rangle\right|^{2} \frac{\left|\left\langle h_{s}(0)\left|j_{0}\right| h_{s^{\prime}}(\vec{q})\right\rangle\right|^{2}}{8 M_{h}^{2} E_{h}(q)} e^{-E_{h}(q)\left(t_{2}-t_{1}\right)} e^{-M_{h}\left(t-\left(t_{2}-t_{1}\right)\right)},
$$

from where the hadron form factor can be extracted in the isospin limit. Note that in practice one can calculate the above quantity for any hadronic state with the same set of propagators. Additionally one has the form factor for all momentum transfers since these follow from the Fourier transform of the density-density correlator.

To demonstrate that this method works in practice we consider the simple case of the pion. The pion as a pseudoscalar meson has only one form factor, which is given by:

$$
\left\langle\pi\left(p_{\mu}^{\prime}\right)\left|J_{\mu}\right| \pi\left(p_{\mu}\right)\right\rangle=\left(p_{\mu}+p_{\mu}^{\prime}\right) F_{\pi}\left(q^{2}\right)
$$

where $q_{\mu}=p_{\mu}^{\prime}-p_{\mu}$. Combining Eq. (4.3) and Eq. (4.2) the form factor $F_{\pi}\left(q^{2}\right)$ can be calculated by dividing the density-density correlator with an appropriate combination of two-point functions:

$$
F_{\pi}^{2}\left(q^{2}\right)=\lim _{\substack{t-t_{2} \gg 1, t_{1} \gg 1, t_{2}-t_{1} \gg 1}} \frac{4 E_{\pi}(q) M_{\pi}}{\left(E_{\pi}(q)+M_{\pi}\right)^{2}} \frac{G^{\pi j^{0} j^{0} \pi}\left(\vec{q} ; t_{1}, t_{2}, t\right) G^{\pi \pi}\left(0, t_{2}-t_{1}\right)}{G^{\pi \pi}\left(\vec{q}, t_{2}-t_{1}\right) G^{\pi \pi}(0, t)} .
$$

The ratio of two-point functions $\left(G^{\pi \pi}\right)$ used in the above equation is the simplest that cancels the unknown overlaps of the interpolating fields with the pion state and the exponential time dependence. However it is not the optimal as far as signal to noise is concerned. Ideally one wants to use two-point functions for the shortest possible time separation since the further the source is from the sink the noisier the propagator becomes. Thus one must find the optimal values for the insertion 


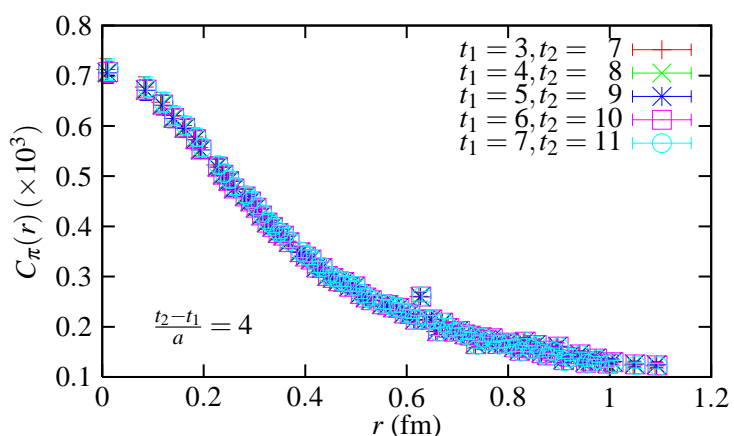

Figure 6: The density-density correlator with the insertion time slices kept fixed at $t_{2}-t_{1}=4$. For this test we only used point to all propagators.

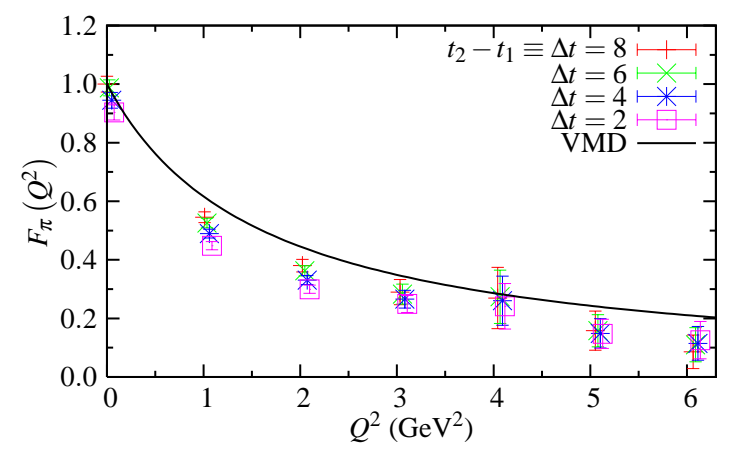

Figure 7: The pion form factor for various values of the time separation between the density insertions. We also compare with the prediction using vector meson dominance (solid line).

times and source-sink separation. The dilemma is that although $t_{2}-t_{1}$ should be large enough to damp any intermediate excited states, the sink-source time separation $t$ should be as small as possible so that the statistical noise on the two-point functions is minimal. Using smearing techniques we can ensure that ground state dominance holds for separations of the density insertions from the source and sink as small as three time slices. This is demonstrated in Fig. 6 where we plot the density-density correlator for the pion with the sink separation fixed at $t=14$ and keeping the time separation between the insertions at a constant value of $t_{2}-t_{1}=4$. We vary the time slice of the density insertions by one starting from $t_{1}=3$ and ending at $t_{1}=7$. It can be seen that the results are identical for $t_{2}, t_{1} \geq 3$ and therefore we can set the first density insertion at $t_{1}=3$ and the second at $t_{2}=11$ three time slices from the sink.

Since $t_{1}=3$ and $t-t_{2}=3$ are the shortest separations we arrange so that the appropriate ratio involves two-point functions in terms of these time separations or time intervals of similar length e.g. $\frac{t_{2}-t_{1}}{2}$ which takes the maximal value of 4 . The optimal ratio is then given by:

$$
F_{\pi}^{2}\left(q^{2}\right)=\lim _{\substack{t-t_{2} \gg 1, t_{1} \gg 1 \\ t_{2}-t_{1} \gg 1}} \frac{4 E_{\pi}(q) M_{\pi}}{\left(E_{\pi}(q)+M_{\pi}\right)^{2}} \frac{G^{\pi j^{0} j^{0} \pi}\left(\vec{q} ; t_{1}, t_{2}, t\right)\left[G_{S L}^{\pi \pi}\left(\vec{q}, t_{1}\right)\right]^{4} G_{S S}^{\pi \pi}\left(0, t_{1}\right)}{G_{S S}^{\pi \pi}\left(0,2 t_{1}\right) G_{S S}^{\pi \pi}\left(0, t-t_{2}\right)\left[G_{S L}^{\pi \pi}\left(\vec{q}, 2 t_{1}\right)\right]^{2}\left[G_{S L}^{\pi \pi}\left(\vec{q}, \frac{t_{2}-t_{1}}{2}\right)\right]^{2}},
$$

where $G_{S S}^{\pi \pi}$ and $G_{S L}^{\pi \pi}$ denote smeared - smeared and smeared - local pion two-point functions respectively. To check for convergence we plot in Fig. $7 F_{\pi}^{2}\left(q^{2}\right)$, given in Eq. (4.5), as a function of $t_{2}-t_{1}$. As can be seen the pion form factor has converged for $t_{2}-t_{1}=8$, which is the maximum even separation that can be achieved on this lattice. On the same plot we compare the form factor to the results obtained assuming vector meson dominance, i.e. taking $F_{\pi}\left(Q^{2}\right)=\frac{1}{1+Q^{2} / m_{\rho}^{2}}$ where $Q^{2}=-q^{2}$ is the Euclidean momentum transfer squared. For $m_{\rho}$ we take the $\rho$ meson mass computed on the lattice which is $m_{\rho}=1.270(8) \mathrm{GeV}$. In Fig. 7 we compare the pion form factor to other recent lattice results extracted using the standard approach of evaluating three-point functions.

Within this method we obtain reliable results up to momentum transfers of $Q^{2} \simeq 6 \mathrm{Gev}^{2}$. The momentum transfers we can extract are limited by the fact that for high momentum transfers the density-density correlator given in Eq. (4.2) becomes too noisy, becoming negative and the square root cannot be taken. 


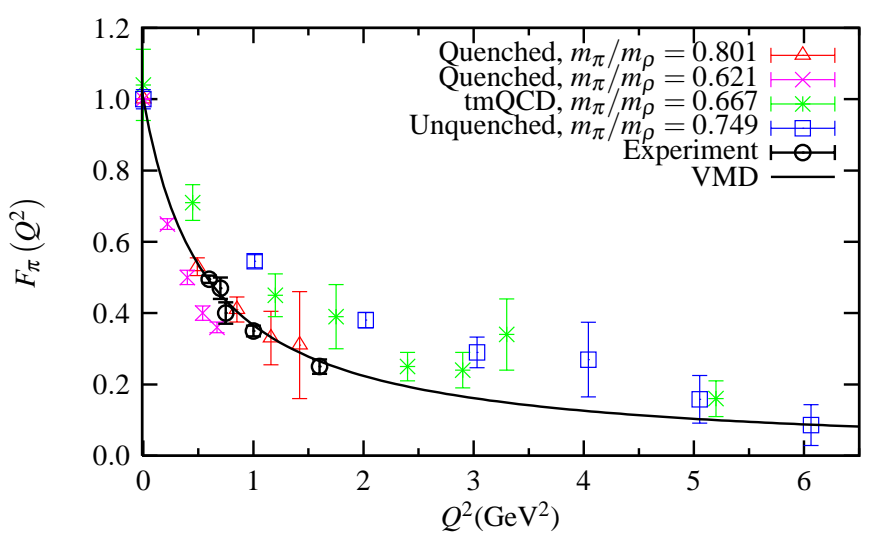

Figure 8: The pion form factor versus $Q^{2}$ with similar $m_{\pi} / m_{\rho}$ ratios. The triangles and crosses show quenched results form Ref. [8], the asterisks are results using twisted mass fermions [9], the squares are the results of this work and the circles denote the experimental results. The solid curve is the results obtained using vector meson dominance with the physical $\rho$ meson mass.

\section{Conclusions}

Stochastic techniques combined with dilution are employed in the evaluation of all to all propagators needed for the exact computation of density-density correlators. The equal time four-point correlators reduce to the wave function squared in the non-relativistic limit and thus provide detailed information on the quark distributions inside the hadrons. In particular the deformation of the hadron can be studied. We clearly detect a deformation in the case of the $\rho$ meson. However, no definite conclusion regarding deformation can be reached for the $\Delta$, at least within these statistics. Another application of density-density correlators is the evaluation of hadron form factors. We apply this method to compute the pion form factor up to $Q^{2} \sim 6 \mathrm{GeV}^{2}$. The results we obtain within this method are comparable to the results extracted from the standard approach using three-point functions.

\section{References}

[1] C. Mertz et al., Phys. Rev. Lett. 86 (2001) 2963, nucl-ex/9902012]; K. Joo et al., Phys. Rev. Lett. 88 (2002) 122001, hep-ex/0110007].

[2] C. Michael and J. Peisa, Phys. Rev. D 58 (1998) 034506, [hep-lat/9802015].

[3] J. Foley et al., Comput. Phys. Commun. 172 (2005) 145, hep-lat/0505023.

[4] C. Alexandrou, Ph. de Forcrand and A. Tsapalis, Phys. Rev. D 66 (2002) 094503, hep-lat/0206026].

[5] SESAM collaboration, Phys. Rev. D 59 (1999) 014509, hep-lat/9806027.

[6] C. Alexandrou et al., Phys. Rev. D 74 (2006) 034508, hep-lat/0605017.

[7] W. Wilcox, Phys. Rev. D 43 (1991) 2443.

[8] F. Bonnet et al., Phys. Rev. D 72 (2005) 054506, hep-lat/0411028.

[9] M. Abdel-Rehim and R. Lewis, Nucl. Phys. (Proc.Suppl.) 140 (2005) 299, [hep-lat/ 0408033 ]. 